Research Article

\title{
Research on Reliability for Servo Turret Based on Vibration Transmission Path System with Stiffness Degradation Model
}

\author{
Li-sha Zhu $\mathbb{D}^{1},{ }^{1}$ Lian-sheng Li $\mathbb{D},{ }^{2}$ Shang-jie Li, ${ }^{3}$ and Shan Wang ${ }^{3}$ \\ ${ }^{1}$ School of Mechanical and Automotive Engineering, Zhaoqing University, Zhaoqing, Guangdong 526061, China \\ ${ }^{2}$ Beijing Institute of Control Engineering, Beijing 100190, China \\ ${ }^{3}$ School of Mechanical Engineering \& Automation, Northeastern University, Shenyang 110819, China
}

Correspondence should be addressed to Lian-sheng Li; liliansheng1981@163.com

Received 2 July 2020; Accepted 28 September 2020; Published 3 November 2020

Academic Editor: Leandro F. F. Miguel

Copyright (C) 2020 Li-sha Zhu et al. This is an open access article distributed under the Creative Commons Attribution License, which permits unrestricted use, distribution, and reproduction in any medium, provided the original work is properly cited.

\begin{abstract}
The stiffness degradation of the servo turret will inevitably lead to accuracy reduction of the cutter head and the tool change. Considering the degradation process of servo turret with stiffness, by introducing the stiffness cumulative damage theory into the vibration differential equation, combined with stochastic finite element method and reliability theory, the mathematical model of the reliability and reliability sensitivity for vibration transmission path system with random parameters was established. Taking a typical power servo turret for example, the reliability and the reliability sensitivity to each random parameter at the mean value with the excitation frequency and time were obtained. The results showed that the shift of the reliability and reliability sensitivity to random parameters with time was caused by the stiffness degradation, the peak value of reliability sensitivity fluctuated with time, and the peak value in the frequency domain at the initial time was not necessarily the maximum value in the time domain. The accuracy of the proposed method was further proved by the Monte Carlo method. Optimizing sensitive parameters could enhance the system stability and effectively prevent the resonance failure caused by the change of the resonance region.
\end{abstract}

\section{Introduction}

At present, there are two kinds of analytical methods for stochastic structural system; one is traditional Monte Carlo simulation [1], and the other is stochastic perturbation [2], second-moment method [3], and stochastic finite element method [4]. All the above methods can effectively solve the problems related to the uncertain vibration transmission path system. However, some of the path parameters are not only random but also time-dependent [5-8]. For example, besides the stochastic characteristic due to factors such as errors and system structure, the stiffness and damping also degrade with time. The change in stiffness complicates the analysis of the vibration transmission path system. In practical engineering, the stiffness of vibration transmission path system will inevitably degrade over time $[5,6,9-11]$. This is an important issue, which cannot be ignored in analyzing the transmission reliability of the transmission path system.

For research on reliability of stochastic structural system, although many scholars have done a lot of work, the research on the reliability of stochastic structural systems with timevarying stiffness is still scarce. Zhao et al. [12] performed sensitivity analysis on power flow of systems with nonlinear and piecewise linear stiffness. Li and Mourelatos [13] proposed a new method for analyzing time-varying reliability for systems with time-varying parameters based on niche genetic algorithms. Ye [14] studied the reliability of a timevarying structural system considering strength attenuation. By constructing the system's full stochastic process reliability model, the reliability expression of the system under the consideration of strength attenuation was obtained. Zhou and Fang [15] studied the system reliability under the change of bearing stiffness of wind turbine drive system caused by 
random wind. At present, many scholars mostly analyzed the time-domain response and response sensitivity of stochastic systems with time-varying parameters. Therefore, the partial reliability results hidden in the frequency domain will be ignored if only the time domain response is analyzed. Similarly, some results hidden in the time domain will also be ignored if only the frequency domain analysis is carried out.

Based on the basic theory of reliability, the theory of miner cumulative damage, and the stochastic finite element, the mathematical expressions of the reliability and reliability-based sensitivity of the vibration transmission path system with random parameters under the consideration of stiffness degradation are derived. The effect of stiffness degradation on system reliability in time and frequency domains is analyzed, which provides an effective method for the reliability analysis of vibration transmission system with variable stiffness.

\section{Vibration Transmission Path System Model with Stiffness Degradation}

Due to the cyclic effect of external excitation, the stiffness of each part of the system will gradually degrade with time. The damage index $D$ model [16] is generally used to describe the degree of stiffness degradation in engineering, $D=0$ means that the stiffness of the structure has not been degraded, and damage index $D=1$ means that the stiffness has been degraded to zero, and the structure has fracture failure. Among them, the Miner theory put forward by Miner-Palmgren is the most widely used stiffness cumulative damage theory [17]. The specific expression of the damage index is

$$
D=A \gamma_{t}^{B} N
$$

It can be seen that the power exponential model of stiffness degradation is

$$
\frac{S}{C}=1-D
$$

In the formula:

$$
\begin{aligned}
& S \text { - Residual stiffness; } \\
& C \text { - Initial damage-free stiffness of the material; } \\
& \text { A, B-Material-dependent constants; } \\
& \gamma_{t} \text {-Cyclic strain amplitude; } \\
& N \text {-Cycle times, during continuous uninterrupted vi- } \\
& \text { bration, it can be expressed as } Q \cdot t, Q \text { is the excitation } \\
& \text { frequency. }
\end{aligned}
$$

Sorting out, the stiffness expression at any moment and the excitation frequency is

$$
S=\left(1-A \gamma_{t}^{B} Q \cdot t\right) C .
$$

It can be seen from the above formula that when the initial stiffness and strain amplitude are given, the stiffness is a function of excitation frequency and time.

In reality, the vibration transmission path system cannot be a completely symmetrical structure, so there will be a moment effect. By analyzing the vibration transmission system of a tool holder system, the vibration form is not only linear vibration, but also swing vibration. Therefore, when considering the relevant parameters of the transmission path, in addition to the stiffness, mass, and damping, the geometric parameters such as the position and shape of the path should also be considered. Because the damping magnitude of the actual structure is small, the influence of damping on the reliability analysis of the structure is ignored here.

As shown in Figure 1, the vibration source and receptor are regarded as rigid structures, its mass is $m_{s}, m_{r}$, and the moment of inertia of the vibration source and receptor around the center of mass is expressed as $I_{s}, I_{r}$. The initial stiffness of the two fixed connections of the vibration source is $k_{s 1}, k_{s 2}$, and the initial stiffness of the fixed connection of the receptor is $k_{r 1}, k_{r 2}$. The position of the fixed source of the vibration source relative to its center of mass is expressed as $y_{s 1}, y_{s 2}$, the position of the receptor fixed junction relative to its centroid is expressed as $y_{r 1}, y_{r 2}$, the location of each transmission path connected by the vibration source is expressed as $y_{s p 1}, y_{s p 2}, y_{s p 3}$, the position where the receptor is connected to each transmission path is expressed as $y_{r p 1}, y_{r p 2}$, $y_{r p 3}$, and the quality of the three transmission paths is expressed as $m_{p 1}, m_{p 2}, m_{p 3}$. There is a problem of concentrated quality in the path, so the stiffness of each path can be divided into two parts, the side next to the vibration source is expressed as $k_{s p 1}, k_{s p 2}, k_{s p 3}$, the stiffness of the side next to the receptor is expressed as $k_{r p 1}, k_{r p 2}, k_{r p 3}$, and the stiffness given above is the initial stiffness of the system. The excitation frequency is expressed as $Q$, the excitation amplitude is $F_{0}$, and the torque is $M_{0}$. It is clear that the system is a 7-DOF (degree of freedom) vibration model, which is vertical and rotational motions of vibration source and receptor at mass center and vertical motion of three transmission paths. The differential equation of motion of a vibration system with linear and rotational coupling motion with variable stiffness is established as [18].

$$
\mathbf{M} \ddot{\mathbf{x}}+\mathbf{K}(Q, t) \mathbf{x}=\mathbf{F}(t) .
$$

In the formula 


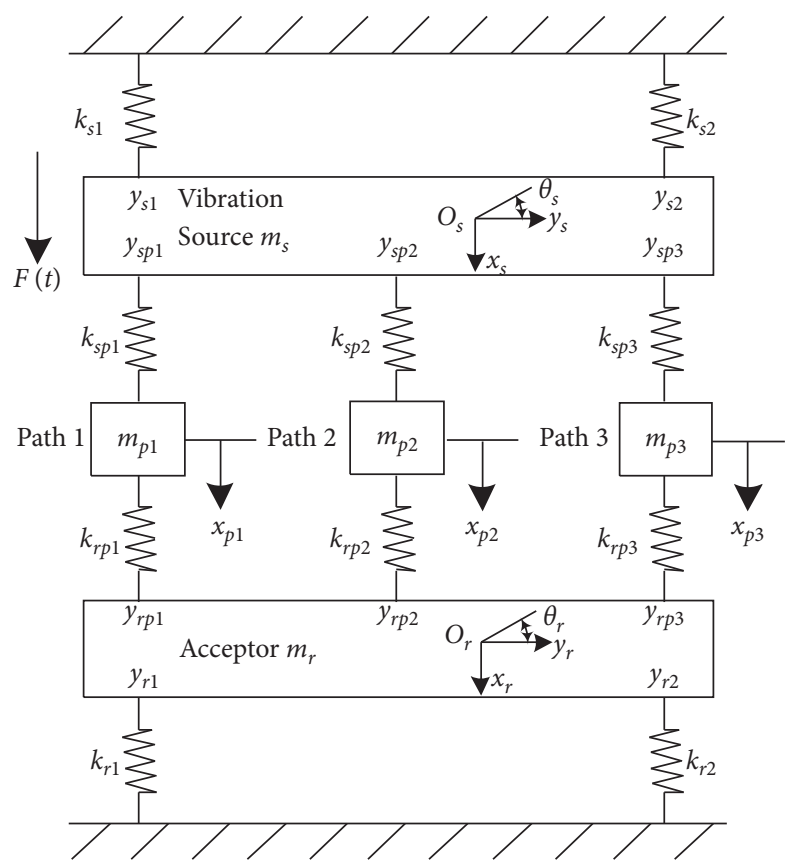

FIgURE 1: Vibration transmission path model of a power servo tool holder.

$$
\begin{aligned}
& \mathbf{M}=\operatorname{diag}\left(\begin{array}{lllllll}
m_{s} & I_{s} & m_{p 1} & m_{p 2} & m_{p 3} & m_{r} & I_{r}
\end{array}\right), \\
& \mathbf{K}(t)=\left[\begin{array}{ccccccc}
\sum D_{S} k_{s n} & -\sum D_{S} k_{s n} y_{s n} & -D_{S} k_{s p 1} & -D_{S} k_{s p 2} & -D_{S} k_{s p 3} & 0 & 0 \\
-\sum D_{S} k_{s n} y_{s n} & \sum D_{S} k_{s n} y_{s n}^{2} & D_{S} k_{s p 1} y_{s p 1} & D_{S} k_{s p 2} y_{s p 2} & D_{S} k_{s p 3} y_{s p 3} & 0 & 0 \\
-D_{S} k_{s p 1} & D_{S} k_{s p 1} y_{s p 1} & D_{S}\left(k_{s p 1}+k_{r p 1}\right) & 0 & 0 & -D_{S} k_{r p 1} & D_{S} k_{r p 1} y_{r p 1} \\
-D_{S} k_{s p 2} & D_{S} k_{s p 2} y_{s p 2} & 0 & D_{S}\left(k_{s p 2}+k_{r p 2}\right) & 0 & -D_{S} k_{r p 2} & D_{S} k_{r p 2} y_{r p 2} \\
-D_{S} k_{s p 3} & D_{S} k_{s p 3} y_{s p 3} & 0 & 0 & D_{S}\left(k_{s p 3}+k_{r p 3}\right) & -D_{S} k_{r p 3} & D_{S} k_{r p 3} y_{r p 3} \\
0 & 0 & -D_{S} k_{r p 1} & -D_{S} k_{r p 2} & -D_{S} k_{r p 3} & \sum D_{S} k_{r n} & -\sum D_{S} k_{r n} y_{r n} \\
0 & 0 & D_{S} k_{r p 1} y_{r p 1} & D_{S} k_{r p 2} y_{r p 2} & D_{S} k_{r p 3} y_{r p 3} & -\sum D_{S} k_{r n} y_{r n} & \sum D_{S} k_{r n} y_{s n}^{2}
\end{array}\right]
\end{aligned}
$$

In the matrix

$$
\begin{aligned}
D_{S} & =1-A \gamma_{t}^{B} Q \cdot t, \\
n & =1,2, p 1, p 2, p 3, \\
\mathbf{x} & =\left[\begin{array}{llllllll}
x_{s} & \theta_{s} & x_{p 1} & x_{p 2} & x_{p 3} & x_{r} & \theta_{r}
\end{array}\right]^{\mathrm{T}}, \\
\mathbf{F}(t) & =\left[\begin{array}{lllllll}
F_{0} \sin (Q \cdot t) & M_{0} \sin (Q \cdot t) & 0 & 0 & 0 & 0 & 0
\end{array}\right]^{\mathrm{T}} .
\end{aligned}
$$

By constructing a random vector, all parameters in the vibration system can be expressed, that is,

$$
\mathbf{b}=\left(\begin{array}{llllllllllllllll}
m_{p 1} & m_{p 2} & m_{p 3} & D_{S} k_{s p 1} & D_{S} k_{s p 2} & D_{S} k_{s p 3} & D_{S} k_{r p 1} & D_{S} k_{r p 2} & D_{S} k_{r p 3} & y_{s p 1} & y_{s p 2} & y_{s p 3} & y_{r p 1} & y_{r p 2} & y_{r p 3}
\end{array}\right)^{\mathrm{T}} .
$$

Here, we know the probability and statistical properties of each element of random vector. According to the above content, the final differential equation of motion of the random parameter vibration system including variable stiffness can be obtained as

$$
\mathbf{M}(\mathbf{b}) \ddot{\mathbf{x}}(\mathbf{b}, t)+\mathbf{K}(\mathbf{b}, Q, t) \mathbf{x}(\mathbf{b}, t)=\mathbf{F}(t) .
$$

\section{Stochastic Finite Element Method for Eigenvalue Analysis of Stochastic Structures}

Since $b_{s}(s=1,2, \ldots, m)$ is a random variable, and the mass and stiffness position parameters are random variables, therefore, the eigenvalues and eigenvectors of the vibration transmission path system are both random variables, which 
are affected by time and excitation frequency. Therefore, the Taylor series expansion of the characteristic equation of the vibration transmission path system can be derived from the relationship between the characteristic value of the random parameter system and the natural frequency of the structure, and the first-order sensitivity of the natural frequency to the random parameter can be derived.

The random eigenvalue problem for this system can be defined as

$$
\mathbf{K} \mathbf{u}_{i}=\omega^{2} \mathbf{M} \mathbf{u}_{i} .
$$

The above formula performs a second-order Taylor expansion at the mean $\overline{\mathbf{b}}$ of the random parameter and compares the same powers. After expanding to the first order, the following recursive equation can be obtained:

$$
\begin{array}{r}
\mathbf{K}(\overline{\mathbf{b}}) \mathbf{u}_{i}(\overline{\mathbf{b}})=\omega_{i}^{2}(\overline{\mathbf{b}}) \mathbf{M}(\overline{\mathbf{b}}) \mathbf{u}_{i}(\overline{\mathbf{b}}), \\
{\left[\left.\frac{\partial \mathbf{K}}{\partial \mathbf{b}^{T}}\right|_{\mathbf{b}=\overline{\mathbf{b}}}-\left.\omega_{i}^{2}(\overline{\mathbf{b}}) \frac{\partial \mathbf{M}}{\partial \mathbf{b}^{T}}\right|_{\mathbf{b}=\overline{\mathbf{b}}}-\left.\frac{\partial \omega_{i}^{2}}{\partial \mathbf{b}^{T}}\right|_{\mathbf{b}=\overline{\mathbf{b}}} \mathbf{M}(\overline{\mathbf{b}})\right] \times \mathbf{u}_{i}(\overline{\mathbf{b}})+\left.\left[\mathbf{K}(\overline{\mathbf{b}})-\omega_{i}^{2}(\overline{\mathbf{b}}) \mathbf{M}(\overline{\mathbf{b}})\right] \frac{\partial \mathbf{u}_{i}}{\partial \mathbf{b}^{T}}\right|_{\mathbf{b}=\overline{\mathbf{b}}}=0 .}
\end{array}
$$

Among them, it is possible to find the expressions of the natural frequency $\bar{\omega}_{i}(Q, t)$ and the eigenvector $\overline{\mathbf{u}}_{i}(Q, t)$ with the time and the excitation frequency as variables when equation (10) is the mean value of the random parameter $\overline{\mathbf{b}}$ through the conventional solution of the characteristic value solution. The first-order sensitivity of the eigenvalue can be obtained by equation (11), and then the first-order sensitivity of the natural frequency of the structure to the mean value $\overline{\mathbf{b}}$ of the random parameter can be obtained. By using $\overline{\mathbf{u}}_{i}^{\mathrm{T}}$ left multiplication (11) and because $\mathbf{K}$ and $\mathbf{M}$ are real symmetric matrices, we can derive

$$
\frac{\partial \bar{\omega}_{i}^{2}}{\partial \mathbf{b}^{\mathrm{T}}} \overline{\mathbf{u}}_{i}^{\mathrm{T}} \overline{\mathbf{M}} \overline{\mathbf{u}}_{i}=\overline{\mathbf{u}}_{i}^{\mathrm{T}}\left(\frac{\partial \overline{\mathbf{K}}}{\partial \mathbf{b}^{\mathrm{T}}}-\bar{\omega}_{i}^{2} \frac{\partial \overline{\mathbf{M}}}{\partial \mathbf{b}^{\mathrm{T}}}\right) \overline{\mathbf{u}}_{i} .
$$

The symbols with " in the formula represent the corresponding values of the random parameters when averaging, and the regularization condition of the feature vector is applied, that is,

$$
\mathbf{u}_{i}^{\mathrm{T}} \mathbf{M} \mathbf{u}_{i}=1 .
$$

A new eigenvector after regularization can be obtained from the above formula, replace equation (13) with equation (12) and further deduce after simplification, and substitute the natural frequency and new eigenvector obtained by equation (10); thus, the first-order sensitivity of natural frequency to the mean value $\overline{\mathbf{b}}$ of random parameter can be obtained

$$
\frac{\partial \bar{\omega}_{i}}{\partial \mathbf{b}^{\mathrm{T}}}=\frac{1}{2 \bar{\omega}_{i}(Q, t)} \times\left[\overline{\mathbf{u}}_{i}^{\mathrm{T}}(Q, t)\left(\frac{\partial \overline{\mathbf{K}}}{\partial \mathbf{b}^{\mathrm{T}}}-\bar{\omega}_{i}^{2}(Q, t) \frac{\partial \overline{\mathbf{M}}}{\partial \mathbf{b}^{\mathrm{T}}}\right) \overline{\mathbf{u}}_{i}(Q, t)\right] .
$$

In the formula, $\overline{\mathbf{u}}_{i}(Q, t)$ is the regularized eigenvector, and the matrix form of formula (14) is

$$
\left[\frac{\partial \bar{\omega}_{i}}{\partial \mathbf{b}^{\mathrm{T}}}\right]=\left[\begin{array}{cccc}
\frac{\partial \bar{\omega}_{1}}{\partial \mathbf{b}_{1}} & \frac{\partial \bar{\omega}_{1}}{\partial \mathbf{b}_{2}} & \cdots & \frac{\partial \bar{\omega}_{1}}{\partial \mathbf{b}_{m}} \\
\frac{\partial \bar{\omega}_{2}}{\partial \mathbf{b}_{1}} & \frac{\partial \bar{\omega}_{2}}{\partial \mathbf{b}_{2}} & \cdots & \frac{\partial \bar{\omega}_{2}}{\partial \mathbf{b}_{m}} \\
\cdots & \cdots & \cdots & \cdots \\
\frac{\partial \bar{\omega}_{n}}{\partial \mathbf{b}_{1}} & \frac{\partial \bar{\omega}_{n}}{\partial \mathbf{b}_{2}} & \cdots & \frac{\partial \bar{\omega}_{n}}{\partial \mathbf{b}_{m}}
\end{array}\right] .
$$

The expression for each element in the matrix is

$$
\frac{\partial \bar{\omega}_{i}}{\partial \mathbf{b}_{s}}=\frac{1}{2 \bar{\omega}_{i}(Q, t)} \times\left[\overline{\mathbf{u}}_{i}^{\mathrm{T}}(Q, t)\left(\frac{\partial \overline{\mathbf{K}}}{\partial \mathbf{b}_{s}}-\bar{\omega}_{i}^{2}(Q, t) \frac{\partial \overline{\mathbf{M}}}{\partial \mathbf{b}_{s}}\right) \overline{\mathbf{u}}_{i}(Q, t)\right] .
$$

The expectation and variance of the natural frequency of this random system can be obtained according to the second-order moment method [19], and its expression is

$$
\begin{aligned}
E\left(\omega_{i}\right) & =\bar{\omega}_{i}, \\
\operatorname{Var}\left(\omega_{i}\right) & =E\left[\omega_{i}-E\left(\omega_{i}\right)\right]^{2}=\left(\frac{\partial \bar{\omega}_{i}}{\partial \mathbf{b}^{\mathrm{T}}}\right)^{[2]} \operatorname{Var}(\mathbf{b}) .
\end{aligned}
$$

The matrix form of the variance can be expressed as 


$$
\operatorname{Var}\left(\omega_{i}\right)=\left[\frac{\partial \bar{\omega}_{i}}{\partial \mathbf{b}^{\mathrm{T}}}\right] \operatorname{Var}(\mathbf{b})\left[\frac{\partial \bar{\omega}_{i}}{\partial \mathbf{b}^{\mathrm{T}}}\right]^{\mathrm{T}}=\left[\frac{\partial \bar{\omega}_{i}}{\partial \mathbf{b}^{\mathrm{T}}}\right]\left[\begin{array}{cccc}
\operatorname{Var}\left(\mathbf{b}_{1}\right) & 0 & \cdots & 0 \\
0 & \operatorname{Var}\left(\mathbf{b}_{2}\right) & \cdots & 0 \\
\ldots & \ldots & \cdots & \cdots \\
0 & 0 & \cdots & \operatorname{Var}\left(\mathbf{b}_{\mathbf{m}}\right)
\end{array}\right]\left[\frac{\partial \bar{\omega}_{i}}{\partial \mathbf{b}^{\mathrm{T}}}\right]^{\mathrm{T}}
$$

In the formula, $\operatorname{Var}(\mathbf{b})$ is the variance matrix of random parameters. When the random parameters are independent of each other, the covariance between the two random parameters is zero.

\section{System Reliability Analysis}

Definitely, the most common failure mode of servo turret is the low accuracy of the cutter head rotation and the tool change. However, the stiffness degradation has a direct effect on the inherent characteristic of servo turret structure, which will finally lead to the low accuracy of servo turret. The most important change of the inherent characteristic is the natural frequency, which will probably cause resonance if the excitation frequency and the natural frequency are very close. The state function of the vibration transmission path system for failure analysis can be defined as the interference theory $[20]$ in reliability as

$$
g_{i j}\left(q_{j}, \omega_{i}\right)=\left|q_{j}-\omega_{i}\right|, \quad i=1,2, \ldots, a ; j=1,2, \ldots, \mathbf{b},
$$

where $q_{j}$ is the $j$-th excitation frequency of the system, and $\omega_{i}$ is the $i$-th order natural frequency in the vibration transmission path system.

According to the relationship between the excitation frequency and the natural frequency, it can be known that the two states of the random structure system are

$$
\begin{cases}g_{i j}\left(q_{j}, \omega_{i}\right)=\left|q_{j}-\omega_{i}\right|>\gamma, & \text { safe, } \\ g_{i j}\left(q_{j}, \omega_{i}\right)=\left|q_{j}-\omega_{i}\right| \leq \gamma, & \text { failure. }\end{cases}
$$

In the formula, $\gamma$ is a prescribed interval where resonance may occur. In reliability analysis, $\gamma$ is usually taken from $5 \%$ to $15 \%$ of the mean value of the natural frequency.

Let $G_{i j}=q_{j}-\omega_{i}$, then the mean and variance of the function $G_{i j}$ are

$$
\begin{aligned}
& \mu_{G_{i j}}=E\left(G_{i j}\right)=\mu_{q_{j}}-\mu_{\omega_{i}}, \\
& \sigma_{G_{i j}}^{2}=\operatorname{Var}\left(G_{i j}\right)=\sigma_{q_{j}}^{2}+\sigma_{\omega_{i}}^{2} .
\end{aligned}
$$

In order to avoid overly complicated reliability analysis of stochastic parameter systems, it can be generally considered that the stochastic parameters of the system follow a normal distribution. When both the excitation frequency and the natural frequency follow a normal distribution and are independent of each other, the quasi-failure probability can be expressed as

$$
P_{f}^{i j}=\Phi\left(\frac{\gamma-\mu_{G_{i j}}}{\sigma_{G_{i j}}}\right)-\Phi\left(\frac{-\gamma-\mu_{G_{i j}}}{\sigma_{G_{i j}}}\right) .
$$

In the formula, $\Phi(\cdot)$ is the standard normal distribution function. It can be known from formula (24) that when any excitation frequency is close to a certain order natural frequency, the entire system will be in a resonance state. Therefore, it can be known that the reliability analysis system under this state function should be a series system, so the quasi failure probability of the entire system can be expressed as

$$
P_{f}=1-\prod_{i=1}^{a} \prod_{j=1}^{\mathbf{b}}\left(1-P_{f}^{i j}\right) .
$$

The transmission reliability of the system is

$$
R=1-P_{f}=\prod_{i=1}^{a} \prod_{j=1}^{\mathbf{b}}\left(1-P_{f}^{i j}\right) .
$$

\section{System Reliability Sensitivity Analysis}

After the reliability analysis is performed on the system, a sensitivity analysis is performed on this basis to determine the order in which the changes in each random parameter affect the system reliability. Defining the partial derivative of reliability to each random parameter as reliability sensitivity [21], the reliability sensitivity of reliability to the mean value of random parameters is

$$
\frac{\mathrm{D} R}{\mathrm{D}^{\mathrm{b}}}=\frac{\partial R}{\partial \beta}\left(\frac{\partial \beta}{\partial \mu_{g}} \frac{\partial \mu_{g}}{\partial \overline{\mathbf{b}}^{\mathrm{T}}}+\frac{\partial \beta}{\partial \sigma_{g}} \frac{\partial \sigma_{g}}{\partial \overline{\mathbf{b}}^{\mathrm{T}}}\right),
$$

where $\beta$ is the reliability index, $\mu_{g}$ is the mean value of the state function, and $\sigma_{g}$ is the standard deviation of the state function.

After deriving from the above formula, the sensitivity expression of the reliability to the random parameter $\overline{\mathbf{b}}^{\mathrm{T}}$ can be obtained as 


$$
\begin{aligned}
\frac{\mathrm{D} R}{\mathrm{D} \overline{\mathbf{b}}^{\mathrm{T}}}= & \sum_{v=1}^{a} \sum_{w=1}^{\mathbf{b}}\left(\prod_{i=1}^{a} \prod_{j=1}^{\mathbf{b}} \frac{1-P_{f}^{i j}}{1-P_{f}^{v w}}\right) \times\left[-\frac{\partial P_{f}^{v w}}{\partial \beta_{1}^{v w}}\left(\frac{\partial \beta_{1}^{v w}}{\partial \mu_{G_{v w}}} \frac{\partial \mu_{G_{v w}}}{\partial \mu_{\omega_{v}}} \frac{\partial \mu_{\omega_{v}}}{\partial \overline{\mathbf{b}}^{\mathrm{T}}}+\frac{\partial \beta_{1}^{v w}}{\partial \gamma} \frac{\partial \gamma}{\partial \mu_{\omega_{v}}} \frac{\partial \mu_{\omega_{v}}}{\partial \overline{\mathbf{b}}^{\mathrm{T}}}\right.\right. \\
& \left.+\frac{\partial \beta_{1}^{v w}}{\partial \sigma_{G_{v w}}} \frac{\partial \sigma_{G_{v w}}}{\partial \overline{\mathbf{b}}^{\mathrm{T}}}\right)-\frac{\partial P_{f}^{v w}}{\partial \beta_{2}^{v w}}\left(\frac{\partial \beta_{2}^{v w}}{\partial \mu_{G_{v w}}} \frac{\partial \mu_{G_{v w}}}{\partial \mu_{\omega_{v}}} \frac{\partial \mu_{\omega_{v}}}{\partial \overline{\mathbf{b}}^{\mathrm{T}}}+\frac{\partial \beta_{2}^{v w}}{\partial \gamma} \frac{\partial \gamma}{\partial \mu_{\omega_{v}}} \frac{\partial \mu_{\omega_{v}}}{\partial \overline{\mathbf{b}}^{\mathrm{T}}}+\frac{\partial \beta_{2}^{v w}}{\partial \sigma_{G_{v w}}} \frac{\partial \sigma_{G_{v w}}}{\partial \overline{\mathbf{b}}^{\mathrm{T}}}\right) .
\end{aligned}
$$

In the formula

$$
\begin{aligned}
& \frac{\partial P_{f}^{v w}}{\partial \beta_{1}^{v \omega}}=\varphi\left(\beta_{1}^{v w}\right)=\frac{1}{\sqrt{2 \pi}} e^{-\left(\left(\beta_{1}^{v w}\right)^{2} / 2\right)}, \\
& \frac{\partial P_{f}^{v w}}{\partial \beta_{2}^{v w}}=-\varphi\left(\beta_{2}^{v w}\right)=-\frac{1}{\sqrt{2 \pi}} e^{-\frac{\left(\beta_{2}^{v w}\right)^{2}}{2}}, \\
& \beta_{1}^{v w}=\frac{E\left(\gamma-G_{v w}\right)}{\sqrt{\operatorname{Var}\left(\gamma-G_{v w}\right)}}=\frac{E\left(\gamma-G_{v w}\right)}{\sigma_{G_{v w}}}, \\
& \beta_{2}^{v w}=\frac{E\left(-\gamma-G_{v w}\right)}{\sqrt{\operatorname{Var}\left(-\gamma-G_{v w}\right)}}=\frac{E\left(-\gamma-G_{v w}\right)}{\sigma_{G_{v w}}}, \\
& \frac{\partial \gamma}{\partial \mathbf{u}_{\omega_{v}}}=0.05 \sim 0.15 \\
& \frac{\partial \beta_{1}^{v w}}{\partial \sigma_{G_{v w}}}=-\frac{\gamma-\mu_{G_{v w}}}{\sigma^{2} G_{v w}} \\
& \frac{\partial \beta_{2}^{v w}}{\partial \sigma_{G_{v w}}}=-\frac{-\gamma-\mu_{G_{v w}}}{\sigma^{2} G_{v w}} \\
& \frac{\partial \sigma_{G_{v w}}}{\partial \overline{\mathbf{b}}^{\mathrm{T}}}=\frac{1}{2 \sigma_{G_{v w}}}\left[\frac{\partial^{2} G_{v w}}{\partial\left(\overline{\mathbf{b}}^{\mathrm{T}}\right)^{2}} \otimes \frac{\partial G_{v w}}{\partial \overline{\mathbf{b}}^{\mathrm{T}}}+\left(\frac{\partial^{2} G_{v w}}{\partial\left(\overline{\mathbf{b}}^{\mathrm{T}}\right)^{2}} \otimes \frac{\partial G_{v w}}{\partial \overline{\mathbf{b}}^{\mathrm{T}}}\right)\right. \\
& \left.\cdot\left(I_{m} \otimes U_{m \times m}\right)\left(I_{m} \otimes \operatorname{Var}(\overline{\mathbf{b}})\right)\right] \text {. }
\end{aligned}
$$

In equation (36), $I_{m}$ is an $m \times m$-dimensional identity matrix, and $U_{m \times m}$ is an $m^{2} \times m^{2}$-dimensional identity matrix.

By substituting the reliability results of equations (26), (14), (22), (29) (36) into (28), the sensitivity $\mathrm{DR} / \mathrm{D} \overline{\mathbf{b}}^{\mathrm{T}}$ of the reliability of the vibration transmission path system to the mean value of random parameters can be obtained.

\section{Example Analysis}

As shown in Figure 1, it is a vibration transmission path model of a dynamic servo tool post system. Its vibration forms include linear vibration and rocking vibration. The parameters of each path of the system are as follows:

$$
\begin{aligned}
& m_{s}=0.7724 \mathrm{~kg} \text {, } \\
& m_{r}=1.0556 \mathrm{~kg} \text {, } \\
& m_{p 1}=0.4 \mathrm{~kg} \text {, } \\
& m_{p 2}=0.5 \mathrm{~kg} \text {, } \\
& m_{p 3}=0.6 \mathrm{~kg} \text {, } \\
& A=9.06 \times 10^{-5} \text {, } \\
& \gamma_{t}=0.0015 \mathrm{~m} \text {, } \\
& B=2.06 \text {, } \\
& \gamma=0.05 \text {, } \\
& k_{s 1}=500 \mathrm{~N} / \mathrm{m} \text {, } \\
& k_{s 2}=500 \mathrm{~N} / \mathrm{m} \text {, } \\
& k_{r 1}=1000 \mathrm{~N} / \mathrm{m} \text {, } \\
& k_{r 2}=1000 \mathrm{~N} / \mathrm{m} \text {, } \\
& k_{s p 1}=k_{r p 1}=800 \mathrm{~N} / \mathrm{m} \text {, } \\
& k_{s p 2}=k_{r p 2}=600 \mathrm{~N} / \mathrm{m} \text {, } \\
& k_{s p 3}=k_{r p 3}=400 \mathrm{~N} / \mathrm{m} \text {, } \\
& I_{r}=7.8772 \times 10^{-3} \mathrm{~kg} \cdot \mathrm{m}^{2} \text {, } \\
& I_{s}=2.5799 \times 10^{-3} \mathrm{~kg} \cdot \mathrm{m}^{2} \text {, } \\
& y_{s 1}=-0.1 \mathrm{~m} \text {, } \\
& y_{s 2}=0.1 \mathrm{~m} \text {, } \\
& y_{r 1}=-0.15 \mathrm{~m} \text {, } \\
& y_{r 2}=0.15 \mathrm{~m} \text {, } \\
& y_{s p 1}=-0.09 \mathrm{~m} \text {, } \\
& y_{s p 2}=0.03 \mathrm{~m} \text {, } \\
& y_{s p 3}=0.1 \mathrm{~m} \text {, } \\
& y_{r p 1}=-0.1 \mathrm{~m} \text {, } \\
& y_{r p 2}=0.02 \mathrm{~m} \text {, } \\
& y_{r p 3}=0.09 \mathrm{~m} \text {. }
\end{aligned}
$$

The stiffness and mass in the transmission path and the external excitation frequency are all independent of the normal distribution, and the variance coefficients are all 0.05. The geometric position parameter also obeys the normal distribution, and its coefficient of variance is 0.005 .

According to equation (26), the reliability of the dynamic servo tool rest system with time and excitation frequency is calculated, as shown in Figure 2. 


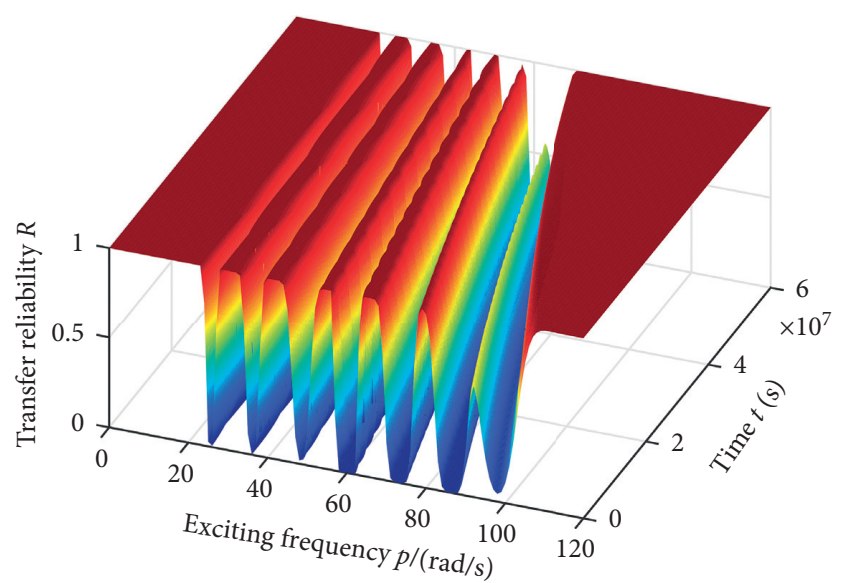

FIgURE 2: Reliability with exciting frequency and time.

According to the eigenvalue analysis of the system, i.e., the calculation of equation (10), it can be obtained that the natural frequencies of each order of the zero-time system at the mean value of the random parameters are

$$
\begin{aligned}
& \bar{\omega}_{1}=25.5760, \\
& \bar{\omega}_{2}=36.1058, \\
& \bar{\omega}_{3}=48.4086, \\
& \bar{\omega}_{4}=59.8445, \\
& \bar{\omega}_{5}=73.0287, \\
& \bar{\omega}_{6}=86.2780, \\
& \bar{\omega}_{7}=98.0629 .
\end{aligned}
$$

It can be seen from Figure 2 that, at time zero, when the excitation frequency is close to the natural frequency of each order of the system, the transmission reliability gradually decreases to 0 . At this time, the system will resonate and be in a state of failure or quasi-failure. When the excitation frequency is far from the natural frequency of each order, the reliability gradually rises to be close to 1 . With the increase of time, the stiffness degradation causes the natural frequency of each order of the system and the variance of the natural frequency to change, thus causing the resonance region to shift. As shown in Figure 2, the troughs gradually gather with time, and the overall interval of resonance in the frequency domain caused by the excitation frequency gradually becomes smaller. Through this analysis, the resonance frequency band can be avoided effectively.

From formula (28), the reliability sensitivity of the transmission reliability of the dynamic servo tool post system to the mean values of various random parameters in the path, including variable stiffness, can be obtained, as shown in Figures 3-17.

From Figures $3-17$, it can be seen that the sensitivity peak value of system transmission reliability to the mean value of each random parameter fluctuates with time due to the influence of stiffness degradation. This phenomenon is due to the stiffness degradation in the time domain, and the system performance is affected by the variable stiffness,

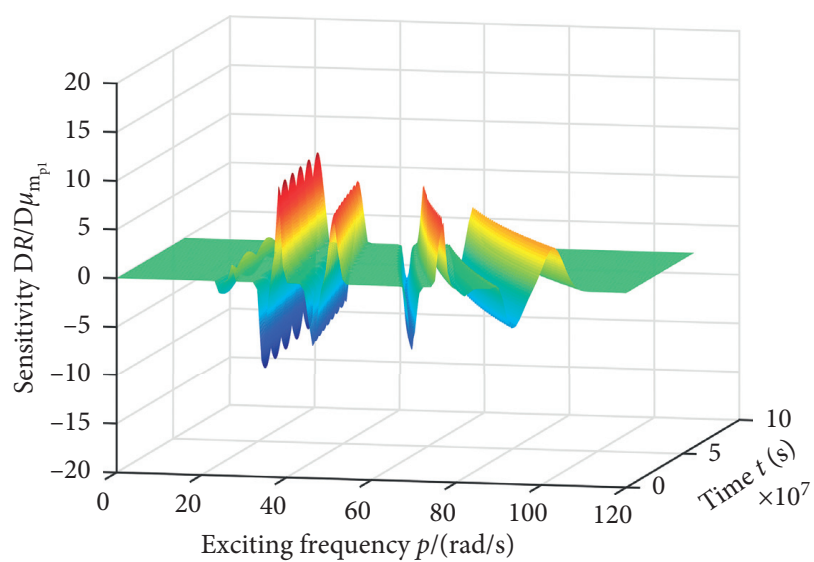

FIGURE 3: Reliability Sensitivity for quality of the first transmission path $m_{p 1}$ at its mean value.

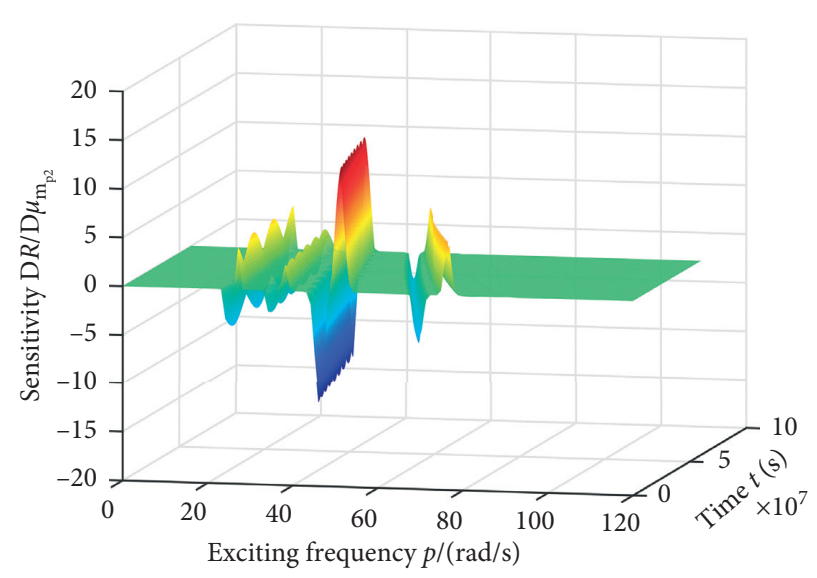

FIgURE 4: Reliability Sensitivity for quality of the second transmission path $m_{p 2}$ at its mean value.

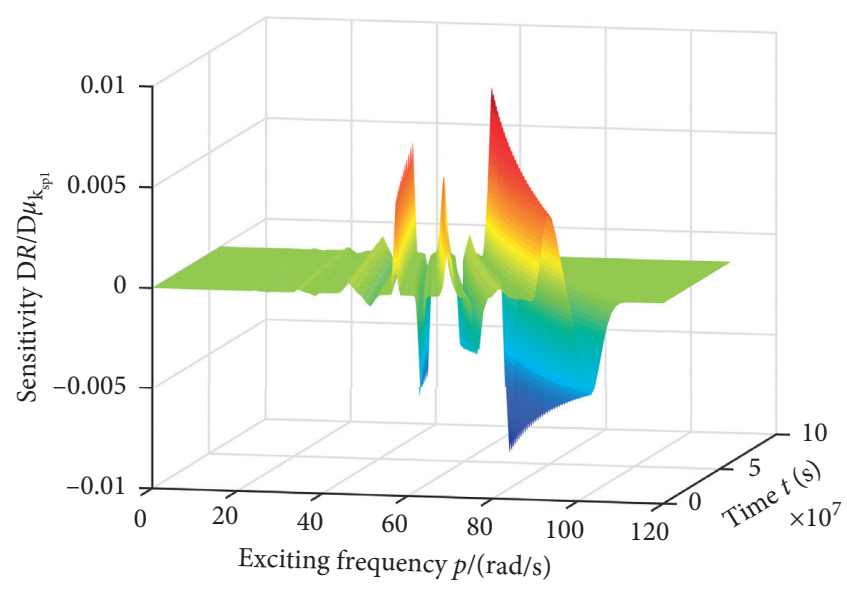

FIGURE 5: Reliability Sensitivity for quality of the third transmission path $m_{p 3}$ at its mean value.

which makes the sensitivity of the system change in the time domain. And as the excitation frequency increases, the frequency of its fluctuations over time also increases. The 


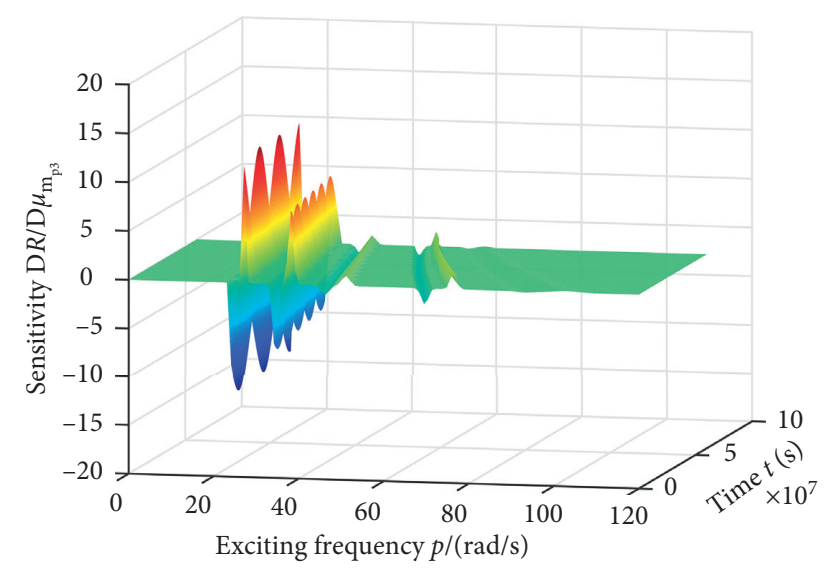

FIgURE 6: Reliability Sensitivity for stiffness of the first path next to the vibration source $k_{s p 1}$ at its mean value.

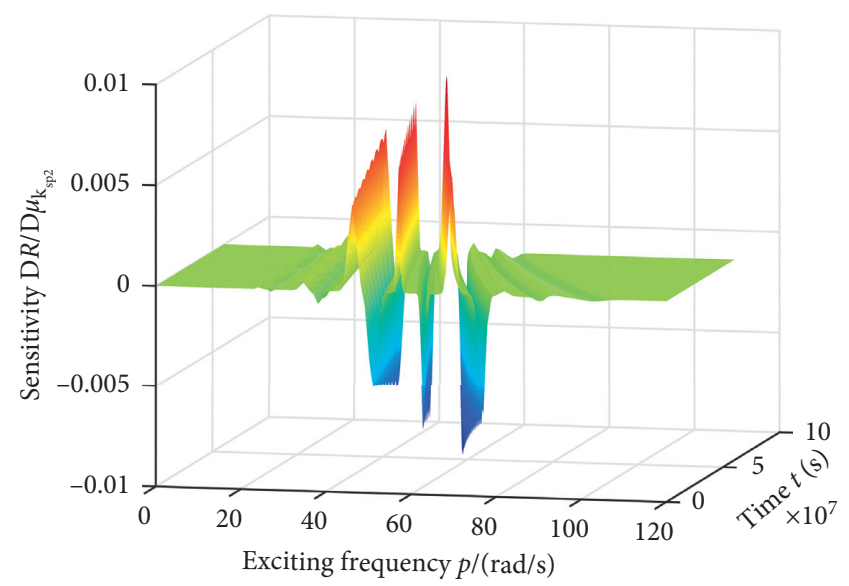

FIgURE 7: Reliability Sensitivity for stiffness of the second path next to the vibration source $k_{s p 2}$ at its mean value.

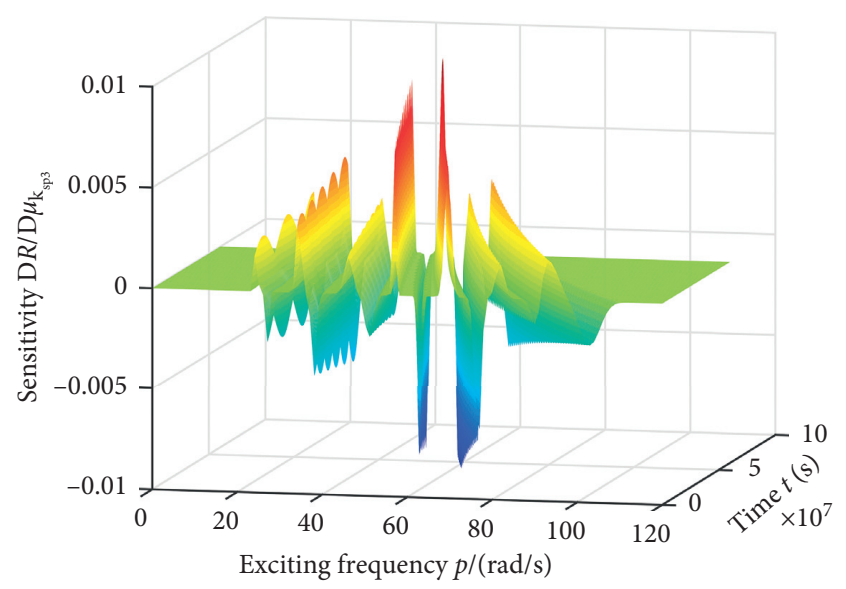

FIGURE 8: Reliability Sensitivity for stiffness of the third path next to the vibration source $k_{s p 3}$ at its mean value.

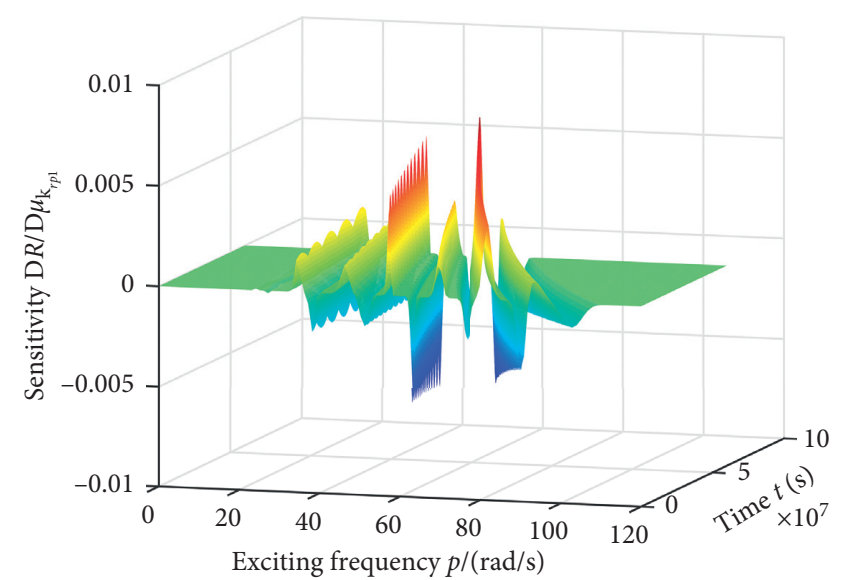

FIGURE 9: Reliability Sensitivity for stiffness of the first path next to the receptor $k_{r p 1}$ at its mean value.

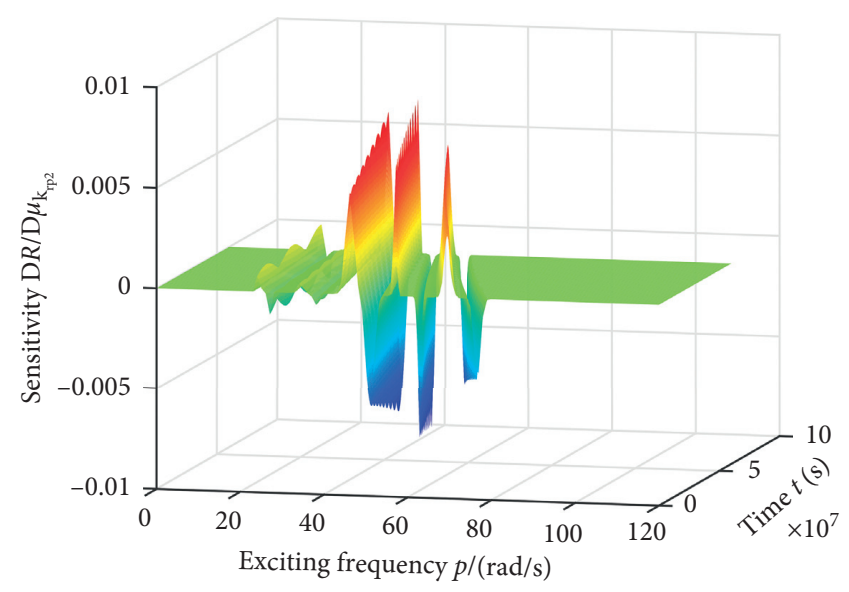

FIgURE 10: Reliability Sensitivity for stiffness of the second path next to the receptor $k_{r p 2}$ at its mean value.

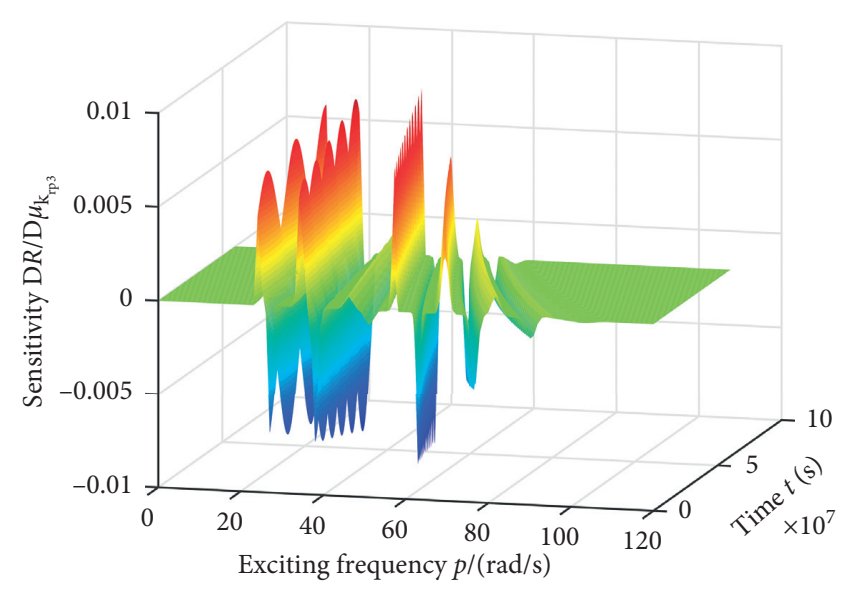

FIgURE 11: Reliability Sensitivity for stiffness of the third path next to the receptor $k_{r p 3}$ at its mean value. 


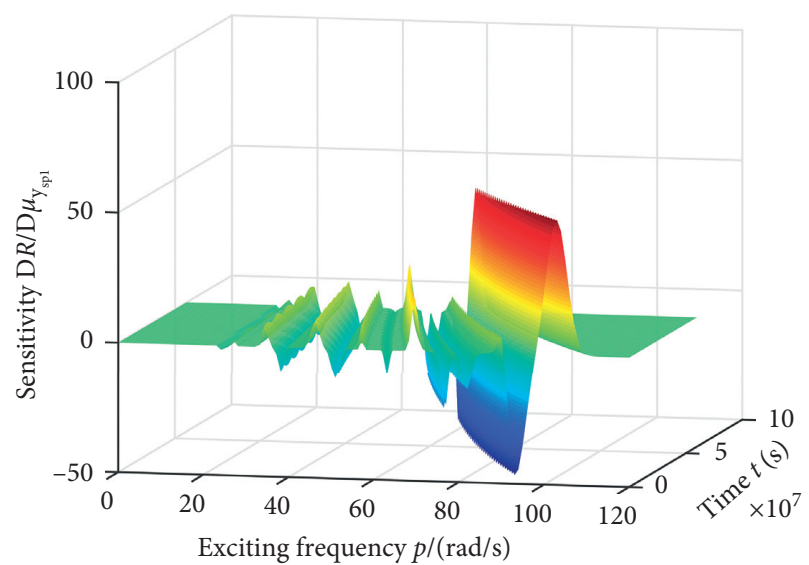

FIgURE 12: Reliability Sensitivity for location of the first path connected by the vibration source $y_{s p 1}$ at its mean value.

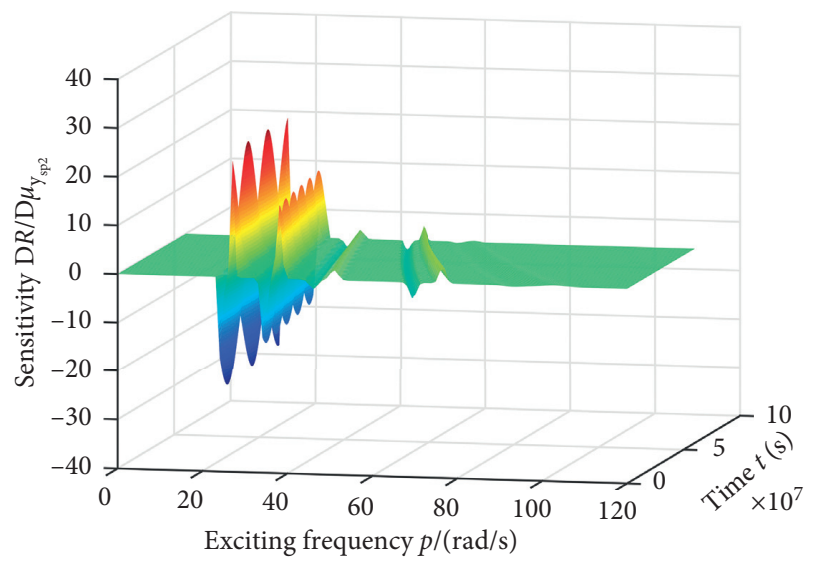

FIGURE 13: Reliability Sensitivity for location of the second path connected by the vibration source $y_{s p 2}$ at its mean value.

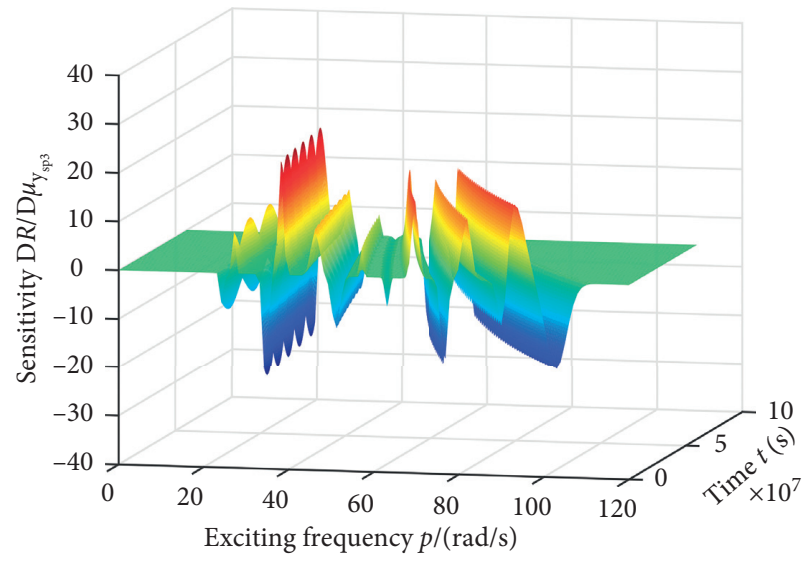

FIgUre 14: Reliability Sensitivity for location of the third path connected by the vibration source $y_{s p 3}$ at its mean value.

form of its fluctuations and the changes in its frequency are affected by the reliability model. When the excitation frequency is larger, the faster the stiffness changes, the faster the

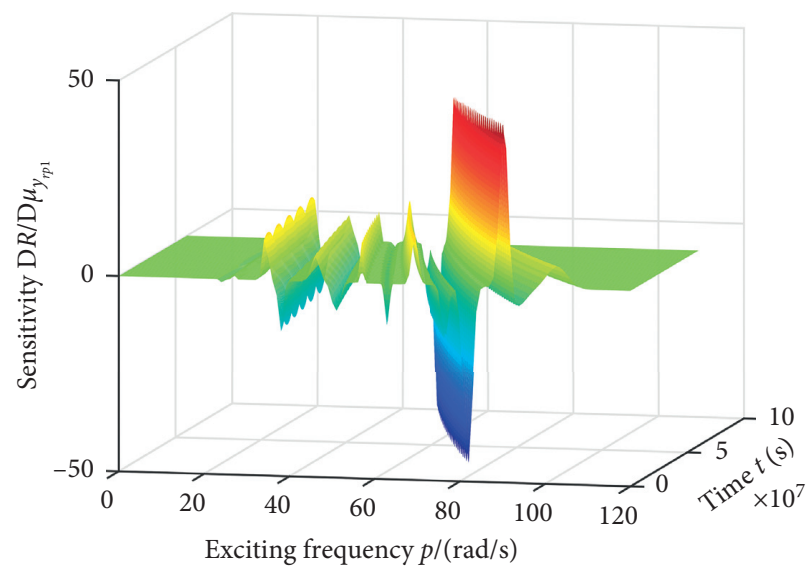

FIGURE 15: Reliability Sensitivity for position where the receptor is connected to the first path $y_{r p 1}$ at its mean value.

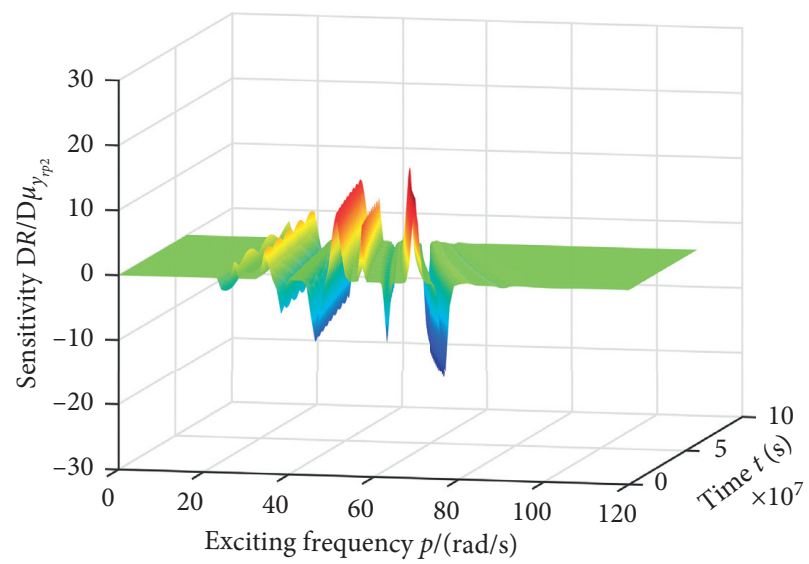

FIGURE 16: Reliability Sensitivity for position where the receptor is connected to the second path $y_{r p 2}$ at its mean value.

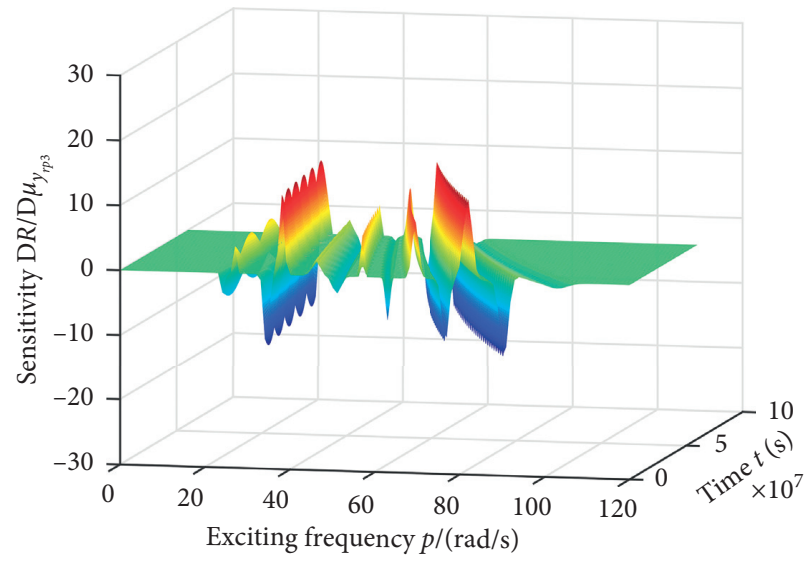

FIGURE 17: Reliability Sensitivity for position where the receptor is connected to the third path $y_{r p 3}$ at its mean value.

sensitivity changes. At the same time, the sensitivity at time zero in the time domain is not necessarily extreme; this shows that the real peak value of sensitivity hidden in the 


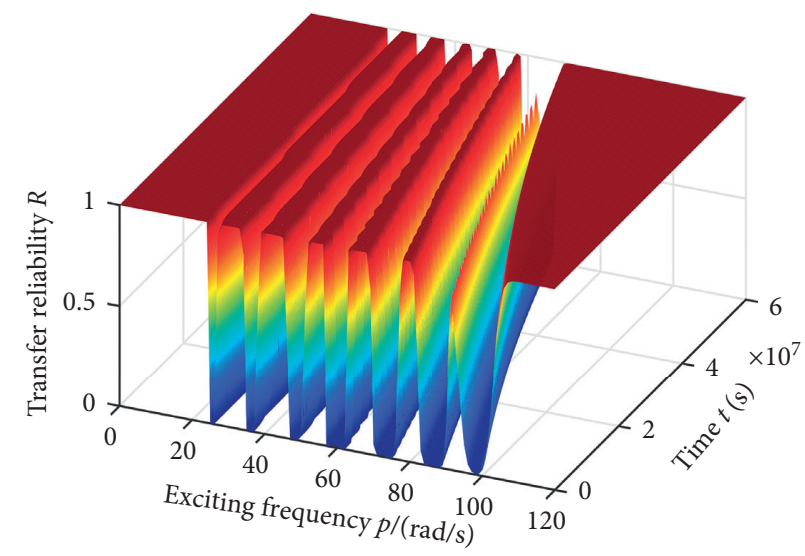

(a)

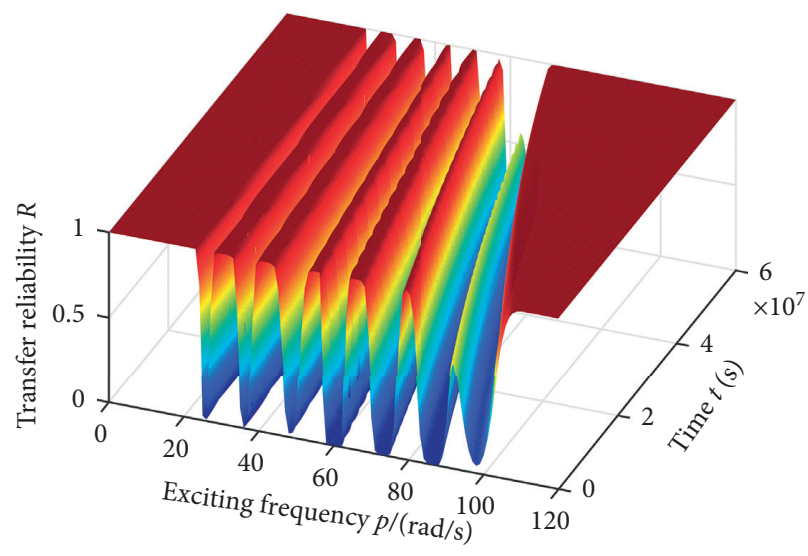

(b)

FIgURE 18: Reliability with exciting frequency and time. (a) Monte Carlo method. (b) Stochastic finite element method.

time domain will be ignored only from the frequency domain analysis. The degradation of stiffness causes the peak value of sensitivity in frequency domain to approach gradually with the increase of time. This shows that the sensitivity of reliability to the mean value of random parameters will not only change with the change of excitation frequency, but also change with time. This provides the basis for the robust optimization of the sensitivity of the reliability to the random parameter mean in the frequency and time domains.

It can be seen from the maximum sensitivity peaks of three transmission paths corresponding to a random parameter that the first transmission path is the most sensitive to the mean value of the reliability, the third is the most sensitive to the mean value of the variable stiffness, and the first is the most sensitive to the mean of the position parameter. Therefore, the sensitivity of reliability to the path parameters can be reduced by optimizing the sensitive parameters of the correlation path such as $m_{p 1}, k_{r p 3}, y_{\mathrm{sp} 1}$ in this special case, which makes the system more stable.

\section{Verification by Monte Carlo Method}

In order to verify the validity and accuracy of the obtained results, a numerical simulation method of reliability analysis is applied to the vibration model of the dynamic servo tool post system; that is, the Monte Carlo method of reliability analysis is used for theoretical verification. The Monte Carlo test method requires a large number of samples. Firstly, the total number of samples of each random parameter at any time and any excitation frequency is set as $n=10000$, and $n$ sample points are randomly generated according to the numerical characteristics of random parameter $\mathbf{b}=\left(m_{p 1}\right.$, $m_{p 2}, m_{p 3}, D_{s} K_{s p 1}, D_{s} K_{s p 2}, D_{s} K_{s p 3}, D_{s} K_{r p 1}, D_{s} K_{r p 2}, D_{s} K_{r p 3}, y_{s p 1}$, $\left.y_{s p 2}, y_{s p 3}, y_{r p 1}, y_{r p 2}, y_{r p 3}\right)^{\mathrm{T}}$. Bring each randomly generated sample point into the characteristic equation and equation (10) to solve the characteristic value. By solving, 10000 groups of natural frequencies can be obtained, which can be substituted into the state function $g_{i j}\left(q_{j}, \omega_{i}\right)=\left|q_{j}-\omega_{i}\right| \quad i=1,2, \ldots, a ; j=1,2, \ldots, \mathbf{b}$, count the number of sample points $n_{f}$ in the failure range $g_{i j}\left(q_{j}, \omega_{i}\right)=\left|q_{j}-\omega_{i}\right| \leq \gamma$, and find the ratio of $n_{f}$ to $n$, which is the failure probability of resonance of the dynamic servo tool post vibration transmission path system at that moment and the excitation frequency. With the help of MATLAB software programming calculation, we finally got the reliability curve of the dynamic servo tool post vibration transmission path system. The comparison with the reliability surface graph calculated by the previous theoretical derivation is as follows:

Comparing with the reliability curve calculated by theoretical derivation, as shown in Figure 18 the reliability curves obtained by the two methods are almost the same. It can be considered that this method has certain accuracy and further proves the validity of the theory. It can be seen that this method is a practical method for solving resonance failure under the condition of stiffness degradation.

\section{Conclusion}

(1) Considering the degradation of stiffness, this paper deduces the mathematical model of the reliability and reliability sensitivity of the vibration transmission path system including random parameters such as variable stiffness, which can be applied to arbitrary vibration systems. It provides an effective theoretical method for analyzing the reliability problem of vibration transmission system including variable stiffness. The damping effect has been ignored as the actual structure in the present paper is small, but it should be considered in the future work if necessary.

(2) By analyzing a dynamic servo tool post system, we obtain the system reliability and reliability sensitivity as a function of the frequency and time of the vibration. The results reflect the influence of stiffness degradation on transmission reliability and reliability sensitivity, and through the theoretical verification of Monte Carlo method, it is proved that this 
method has certain accuracy. By analyzing the changes of the reliability and reliability sensitivity in the time domain direction and the frequency domain direction caused by the degradation of stiffness, the transmission path system can be effectively optimized, which is of great significance for solving practical problems in engineering.

\section{Data Availability}

Some or all data, models, or code generated or used during the study are available from the corresponding author by request.

\section{Conflicts of Interest}

The authors declare that they have no conflicts of interest.

\section{Acknowledgments}

This study is supported by the National Natural Science Foundation of China (Nos. 51975511 and 51475086), Fundamental Research Funds for the Central Universities of China (No. N2023023), and CAST-BISEE Foundation (No. CAST-BISEE2019-019).

\section{References}

[1] E. Zio, The Monte Carlo Simulation Method for System Reliability and Risk Analysis, Springer, London, UK, 2013.

[2] H. Lu and Z. Zhu, "A copula-based method for reliability sensitivity analysis of structural system with correlated failure modes," Eksploatacja i Niezawodnosc-Maintenance and Reliability, vol. 17, no. 3, pp. 450-456, 2015.

[3] L. Yu, L. Meng, K. Liu, and Y. Zhang, "Chatter reliability of milling system based on first-order second-moment method," International Journal of Advanced Manufacturing Technology, vol. 87, pp. 801-809, 2016.

[4] L. Huang, H. Shuai, C. Tao, and Y. Liang, "The finite element method for the reliability analysis of lining structures based on Monte Carlo stochastic," Cluster Computing, vol. 20, no. 4, pp. 1-13, 2017.

[5] M. Fan, Z. Zeng, E. Zio, and R. Kang, "Modeling dependent competing failure processes with degradation-shock dependence," Reliability Engineering \& System Safety, vol. 165, pp. 422-430, 2017.

[6] S. Hao and J. Yang, "Reliability analysis for dependent competing failure processes with changing degradation rate and hard failure threshold levels," Computers \& Industrial Engineering, vol. 118, pp. 340-351, 2018.

[7] D. Kong, N. Balakrishnan, and L. Cui, "Two-phase degradation process model with abrupt jump at change point governed by Wiener process," IEEE Transactions on Reliability, vol. 66, no. 4, pp. 1345-1360, 2017.

[8] H. Peng, Q. Feng, and D. W. Coit, "Reliability and maintenance modeling for systems subject to multiple dependent competing failure processes," IIE Transactions, vol. 43, no. 1, pp. 12-22, 2010.

[9] H. Gao, L. Cui, and Q. Qiu, "Reliability modeling for degradation-shock dependence systems with multiple species of shocks," Reliability Engineering \& System Safety, vol. 185, pp. 133-143, 2018.
[10] S. Hao, J. Yang, X. Ma, and Y. Zhao, "Reliability modeling for mutually dependent competing failure processes due to degradation and random shocks," Applied Mathematical Modelling, vol. 51, pp. 232-249, 2017.

[11] S. Song, D. W. Coit, and Q. Feng, "Reliability analysis of multiple-component series systems subject to hard and soft failures with dependent shock effects," IIE Transactions, vol. 48 , no. 8, pp. 720-735, 2016.

[12] Q. Zhao, Y. Zhang, and J. Zhao, "Sensitivity analysis of nonlinear stiffness vibration transfer path system," Journal of Northeastern University (Natural Science), vol. 30, no. 8, pp. 1174-1177, 2009.

[13] J. Li and Z. P. Mourelatos, "Time-dependent reliability estimation for dynamic problems using a niching genetic algorithm," Journal of Mechanical Design, vol. 131, no. 7, 2009.

[14] H. Ye, Stochastic Process Model for Time-Varying Reliability Analysis of Engineering Structures, Fujian Normal University, Fuzhou, China, 2015.

[15] Z. Zhou and X. U. Fang, "Dynamic reliability analysis of gear transmission system of wind turbine considering strength degradation and dependent failure," Journal of Mechanical Engineering, vol. 52, no. 11, pp. 80-87, 2016.

[16] B. Yu, Study on Dynamics and Fatigue Characteristics of Accessory Drive System under Impact Load, Shenyang University of Technology, Shenyang, China, 2017.

[17] X. F. Liu, Z. Wu, M. Song, and M. Zheng, "Based on the theory of the miner fatigue damage of fuzziness analysis and mathematical modeling," Applied Mechanics and Materials, vol. 437, pp. 124-128, 2013.

[18] W. Zhao, Y. Zhang, and N. Zhou, "Parametric sensitivity of vibration transfer path system in frequency domain," Chinese Journal of Construction Machinery, vol. 13, no. 6, pp. 475-479, 2015.

[19] X. Huang, Y. Li, Y. Zhang, and X. Zhang, "A new direct second-order reliability analysis method," Applied Mathematical Modelling, vol. 55, pp. 68-80, 2018.

[20] W. Zhao, Research and Application of Transmitting of Mechanical Vibration Transmission Path System, Northeastern University, Shenyang, China, 2012.

[21] X. Wang, M. Chang, H. Zhang, and C. Lv, "Reliability and sensitivity design of indexing accuracy of end servo of power servo tool holder," Journal of Northeastern University (Natural Science), vol. 38, no. 6, pp. 834-838, 2017. 